\title{
Phenotypic Assays for Detection of AmpC and MBL Producers among the Clinical Isolates of Multi Drug Resistant Pseudomonas aeruginosa
}

\author{
Sarita Manandhar ${ }^{1^{*}}$, Sarashwoti Adhikari ${ }^{1}$, Sujeeb Rajbhandari ${ }^{2}$ \\ ${ }^{1}$ Tri Chandra Multiple College, Ghantaghar, Kathmandu, Nepal \\ ${ }^{2}$ Shahid Gangalal National Heart Center, Bansbari, Kathmandu, Nepal
}

*Corresponding author: Sarita Manandhar, Tri Chandra Multiple College, Ghantaghar, Kathmandu, Nepal Email: sarita111@gmail.com

\begin{abstract}
Objectives: In order to determine the prevalence of multi-drug resistance along with AmpC and metallo- $\beta$-lactamase producing $P$. aeruginosa, a six month cross-sectional study was carried out at Shahid Gangalal National Heart Center.
\end{abstract}

Methods: A total of 756 clinical specimens were analyzed for bacteriological profile. The bacterial isolates were identified by cultural and biochemical techniques. Antibiotic susceptibility testing of the isolates was performed by Kirby-Bauer disc diffusion method. MDR isolates were screened and tested for MBL and AmpC production. Ceftazidime resistant isolates were tested for MBL and Cefoxitin resistant isolates for AmpC.

Results: Among all the clinical samples analyzed, P. aeruginosa was detected in 75 samples (9.92\%). Antibiotic susceptibility testing showed Imipenem as the most effective drug with susceptibility of $76 \%$ followed by Piperacillin-Tazobactam (74.7\%) and Piperacillin (41.3\%). Out of 75 P. aeruginosa isolates, $53(70.6 \%)$ of them were found to be resistant to at least three out of four anti-pseudomonal agents, thus were considered as MDR. Out of 53 multi-drug resistant $P$. aeruginosa (MDRPA), all were resistant to ceftazidime whereas $85 \%$ (45/53) were resistant to cefoxitin. Out of 53 isolates, 11 $(20.75 \%)$ showed positive result for MBL. Similarly, 7 out of 45 i.e. $13.2 \%$ were found to be AmpC producers.

Conclusion: This study signified the high prevalence of MDRPA which is an alarming rate. Also multiple $\beta$-lactamase producing $P$. aeruginosa were detected which can further complicate the treatment options. Regular monitoring of antibiotic susceptibility and rational use of antibiotics would be helpful in eliminating the outbreaks of multiple $\beta$-lactamase producing MDRPA.

Key words: $P$. aeruginosa, $\beta$-lactams, MDRPA, AmpC, MBL

\section{INTRODUCTION}

Pseudomonas aeruginosa is an increasingly prevalent opportunistic human pathogen and the most common gram-negative bacterium found in nosocomial infections (Kalaivani 2011). Most distinguishing feature of this organism is the capability to confer resistance to several classes of antibiotics using distinctive mechanisms (Kapoor et al. 2011; Tian et al. 2011). Though carbapenems remain one of the best drugs to treat infections caused by P. aeruginosa, increasing usage of these drugs and other expanded-spectrum antibiotics has resulted in the development of carbapenem resistant
P. aeruginosa creating therapeutic problem (Yousefi et al. 2010; Martinez et al. 2011). Aztreonam is the only $\beta$-lactam antibiotic that is saved from the activity of $\beta$-lactamases (Tsakris et al. 2009). The common form of resistance is mediated by lack of drug penetration and/or carbapenem hyrolysing $\beta$-lactamase enzymes including the metallo- $\beta$-lactamases (MBL) (Shanthi and Sekar 2009; Ishii et al. 2010). Metallo- $\beta$-lactamases are a diverse set of enzymes having zinc ion at the active site that catalyze the hydrolysis of a broad range of $\beta$-lactam drugs (including carbapenems) with the exception of monobactams. An increasing prevalence of carbapenem 
resistance mediated by acquired metallo- $\beta$-lactamases (MBLs) is being reported, particularly for P. aeruginosa clinical isolates in several countries. The resistance may spread rapidly to various species of gram-negative bacilli, as the MBL genes reside in mobile gene cassettes inserted in integrons. The production of metallo- $\beta$ lactamases by bacteria is becoming a serious threat to the clinical community because these enzymes are responsible for the development of antibiotic resistance to the commonly employed $\beta$-lactam antibiotics.

Ambler class C, confer resistance to cephamycins, narrow, expanded and broad-spectrum cephalosporins, Aztreonam and $\beta$-lactam/ $\beta$-lactamase inhibitor combination. The enzyme may be chromosomal or plasmid encoded. Although these two enzymes have similar substrate profile, the only difference is chromosomal AmpC are inducible whereas plasmid mediated AmpC are uninducible.

Despite the discovery of AmpC and Metallo- $\beta$ lactamases at least a decade ago, there remains a low level of awareness of their importance and many clinical laboratories have problems in detecting AmpC and metallo- $\beta$-lactamase. Failure to detect these enzymes has contributed to their uncontrolled spread and sometimes to therapeutic failures (Singhal et al. 2005).

\section{MATERIALS AND METHODS}

This hospital based cross sectional study was carried out fromApril 2014 to September 2014 in the Microbiology laboratory of Shahid Gangalal National Heart Center. A total of 756 different samples incuding blood, sputum, urine, pus, endo-tracheal (ET) secretion, wound swab, bed sore swab, Central Venous Pressure (CVP) tip, nasal secretion and oral secretion were collected from the patient and processed following standard laboratory techniques. The samples were inoculated on Blood agar and MacConkey agar and incubated at $37^{\circ} \mathrm{C}$ for 24 hours. For the identification of Pseudomonas spp., typical non-fermenting colonies from MacConkey agar were further processed via gram staining and other biochemical tests. P. aeruginosa was identified on the basis of various characteristics such as positive catalase and oxidase test, motile, mannitol non-fermenter, growth at $42^{\circ} \mathrm{C}$, oxidative in Hugh and Leifson's medium, indole negative, methyl red/voges proskauer test negative, citrate positive, Alk/no change, $\mathrm{H}_{2} \mathrm{~S}$ negative, and gas negative in TSIA medium, urease negative and growth on cetrimide agar with production of pigments and fruity odour. Antibiotic susceptibility testing of isolates was assessed as per CLSI (2013) guidelines following modified Kirby-Bauer disc diffusion method. For differentiation of MDRPA isolates, 4 different antipseudomonal classes i.e. carbapenem (Imipenem), cephalosporin (Ceftazidime), fluoroquinolone (Ciprofloxacin) and aminoglycoside (Amikacin) were used.

Only those MDR isolates resistant to Ceftazidime were tested for metallo- $\beta$-lactamase enzyme by two methods. Combined disc testwere done by placing two $10 \mu \mathrm{g}$ Imipenem discs $25 \mathrm{~mm}$ apart on the surface of Mueller Hinton Agar plates inoculated with a bacterial suspension equivalent to $0.5 \mathrm{McF}$ arland standard. One of the disc was loaded with $10 \mu 1$ of $0.5 \mathrm{M}$ EDTA to obtain desired concentration of $750 \mu$ g. After 24 hours of incubation at $37^{\circ} \mathrm{C}$, an increase of $\geq 7 \mathrm{~mm}$ in the zone diameter of EDTA containing Imipenem disc compared to Imipenem disc alone was considered to be a positive test for the presence of an MBL. In double disc synergy test, a10 $\mu \mathrm{g}$ Imipenem disc was placed $20 \mathrm{~mm}$ centre to centre from a blank disc containing $10 \mu \mathrm{l}$ of $0.5 \mathrm{M}$ EDTA on the surface of Mueller Hinton Agar inoculated with test organism. After incubation at $37^{\circ} \mathrm{C}$ overnight, an increase of $\geq 7 \mathrm{~mm}$ in the zone diameter of blank disc containing EDTA compared to Imipenem disc was considered to be a positive test for the presence of an MBL.

AmpC $\beta$-lactamase production was screened by Cefoxitin disc diffusion test. Isolates showing inhibition zone diameter $<18 \mathrm{~mm}$ (screening positive) were further subjected to disc antagonism test for inducible AmpC enzyme and AmpC disc test for the detection of plasmid AmpC Blactamases.

In disc antagonism test, $0.5 \mathrm{McF}$ arland suspension of test isolate was swabbed on MuellerHinton agar plate and Cefotaxime $(30 \mu \mathrm{g})$ and Cefoxitin $(30 \mu \mathrm{g})$ discs were placed $20 \mathrm{~mm}$ apart from centre to centre. After incubation at $37^{\circ} \mathrm{C}$ overnight, isolates showing blunting of the Cefotaxime zone of inhibition adjacent to the Cefoxitin disc were considered positive for inducible AmpC $\beta$-lactamase.

In AmpC disc test, a lawn culture of 0.5 McFarland suspensions of Escherichia coli ATCC 25922 was inoculated on surface of MuellerHinton agar plate. Sterile disc $(6 \mathrm{~mm})$ was moistened with sterile saline 
$(20 \mu \mathrm{l})$ and inoculated with several colonies of test organism. The inoculated disc was then placed beside a $30 \mu \mathrm{g}$ Cefoxitin disc, almost touching it, on the inoculated plate. After overnight incubation at $37^{\circ} \mathrm{C}$, positive test was interpreted as a flattening or indentation of the Cefoxitin inhibition zone in the vicinity of the test disc and negative test as absence of distortion.

\section{RESULTS}

Out of 756 samples investigated, significant growth was observed in 242 of them, among which 75 clinical isolates were identified as $P$. aeruginosa by standard microbiological procedures showing prevalence of 9.92\% (Figure 1).



Figure 1: Prevalence of $P$. aeruginosa among clinical samples

Among different type of clinical samples analyzed, maximum number of $P$. aeruginosa was isolated from endo-tracheal (ET) secretions (69.3\%) followed by the category others (swabs, oral and nasal secretions) $(12 \%)$, sputum $(8 \%)$, urine $(5.3 \%)$, blood $(4 \%)$ and pus (1.3\%) (Figure 2).

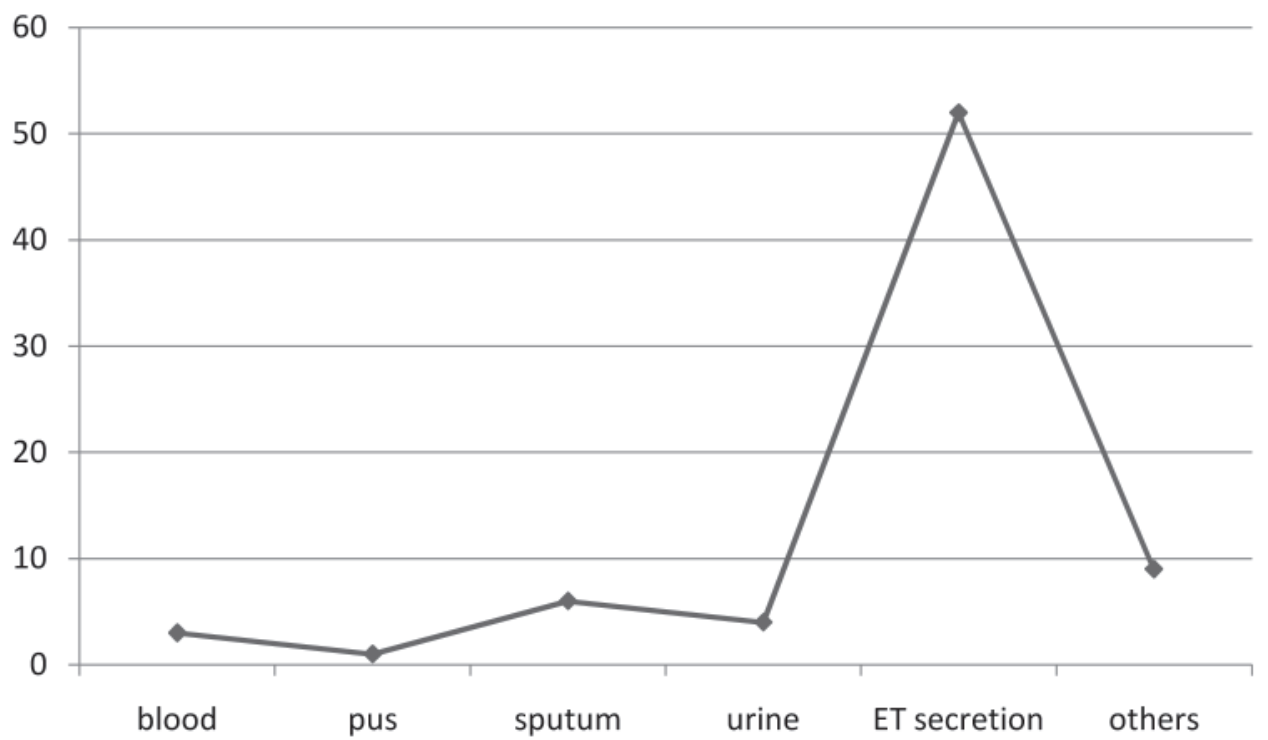

Figure 2: Distribution of $P$. aeruginosa among different specimens

Majority of the isolates were found to be resistant to most of the antibiotics tested. They showed highest resistance to Ceftazidime ( $88 \%$ ) followed by Gentamicin (84\%), Ofloxacin (82.7\%) and Ciprofloxacin (78.7\%).
Imipenem was found to be the most effective drug with susceptibility of $76 \%$ closely followed by PiperacillinTazobactam to which $74.7 \%$ of the organisms were susceptible (Figure 3). 


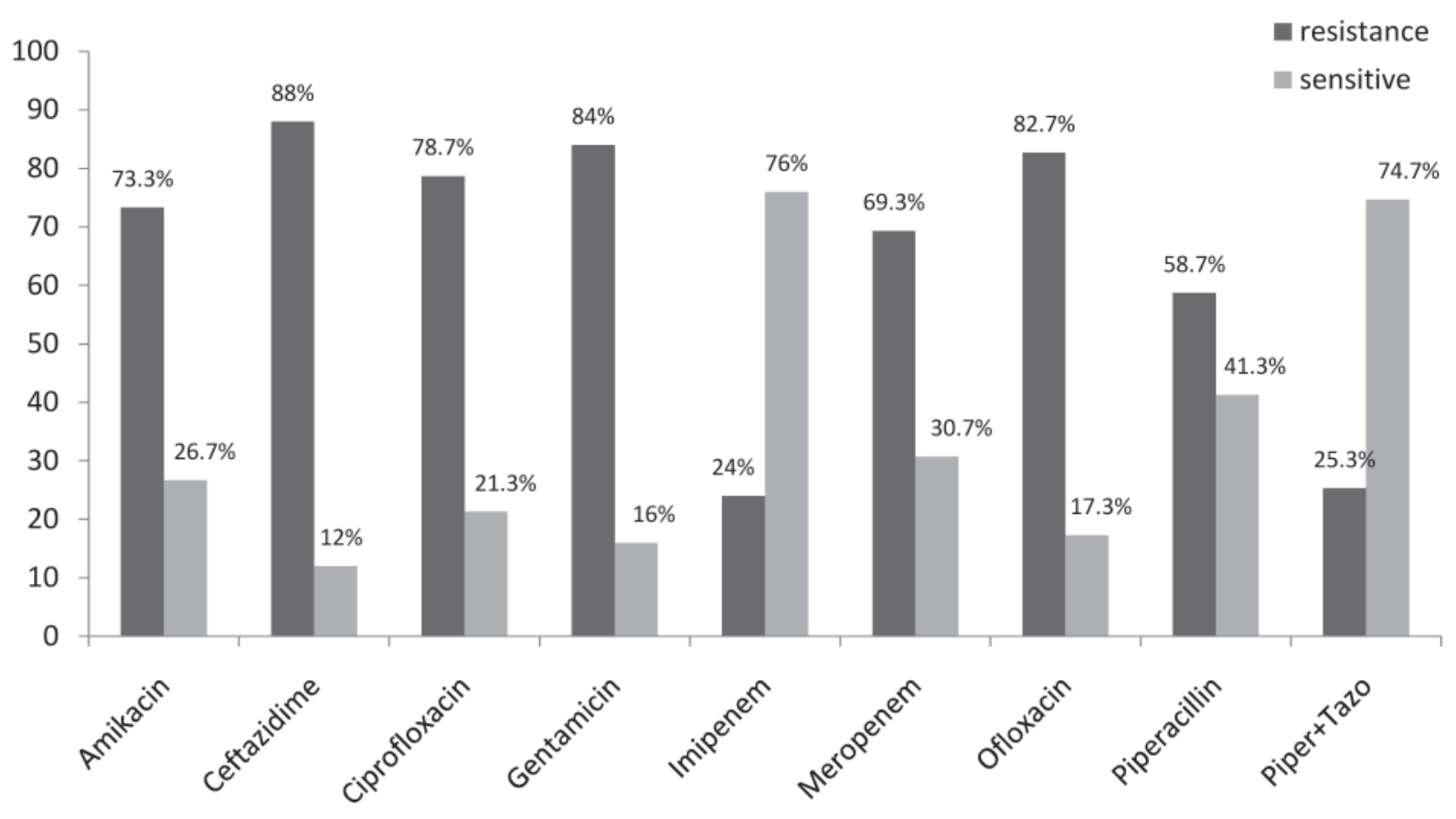

Figure 3: Antibiotic susceptibility pattern of $P$. aeruginosa

Out of 75 isolates, $70.6 \%$ of them were found to be multidrug resistant $P$. aeruginosa (MDRPA). Out of 68 isolates from inpatient, $72.05 \%$ showed multi-drug resistance whereas in case of outpatient, out of 7 isolates, 4 of them were MDR. Altogether out of 53 MDRPA, 92.45\% were from inpatient whereas $7.54 \%$ were from outpatient. There was no significant association between the MDR occurrence and the type of patient $(p>0.05)$ (Table 1$)$.

Table 1: Distribution of multi drug resistant $P$. aeruginosa

\begin{tabular}{lcccc}
\hline \multirow{2}{*}{ Type of strains } & \multicolumn{2}{c}{ Type of patient } & Total (\%) & p-value \\
\cline { 2 - 3 } & Inpatient & Outpatient & & \\
\hline MDR & $49(92.45 \%)$ & $4(7.54 \%)$ & $53(70.6 \%)$ & 0.41 \\
Non-MDR & 19 & 3 & $22(29.3 \%)$ & \\
\hline Total & 68 & 7 & $75(100 \%)$ & \\
\hline
\end{tabular}

Among different types of clinical samples analyzed during the study, maximum no. of MDR isolates were observed in endotracheal (ET) secretion (73.58\%) followed by the category others $(9.43 \%)$, sputum $(7.54 \%)$, and urine
(5.66\%). Only $1.88 \%$ of the total MDR isolates were isolated from both blood and pus individually. The association between the MDR occurrence and specimen was found to be statistically insignificant $(\mathrm{p}>0.05)$ (Table 2).

Table 2: Distribution of MDRPA among various samples

\begin{tabular}{lcccc}
\hline \multicolumn{1}{c}{ Type of Specimen } & No. of $P$. aeruginosa & No. of MDR isolates & \% of MDR isolates & P-value \\
\hline Blood & 3 & 1 & 1.88 \\
Pus & 1 & 1 & 1.88 \\
Sputum & 6 & 4 & 7.54 \\
Urine & 4 & 3 & 5.66 \\
ET secretion & 52 & 39 & 73.58 \\
Others & 9 & 5 & 9.43 \\
\hline Total & 75 & 53 & 100 \\
\hline
\end{tabular}


Ceftazidime resistant isolates were tested for MBL production. In this study, out of 53 MDR isolates, all were resistant to Ceftazidime. Altogether 11 (20.75\%) of them gave positive results for MBL by combined disc test and double disc synergy test (Figure 4).

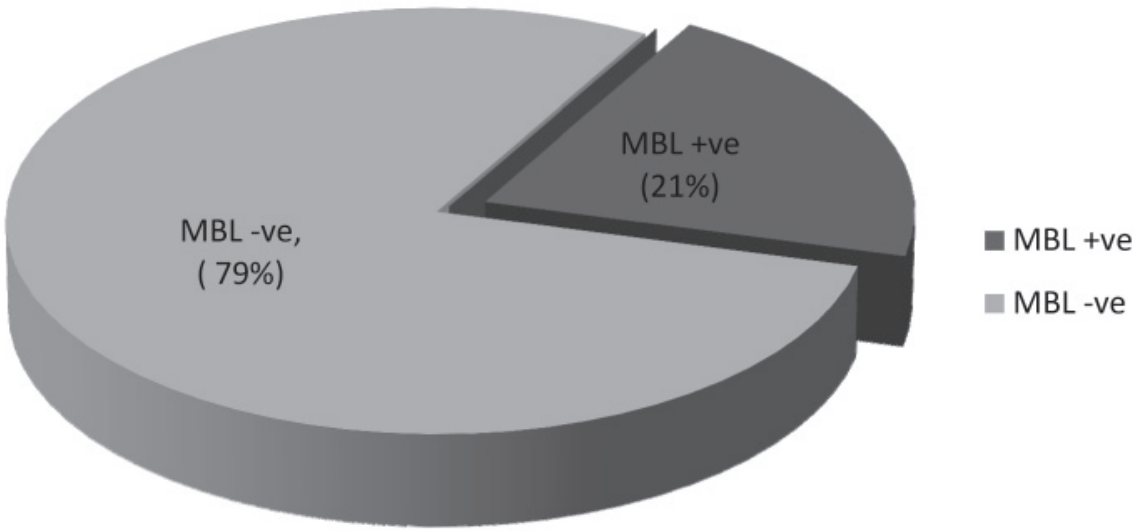

Figure 4: MBL production among MDR $P$. aeruginosa

Among two methods used for the detection of metallo$\beta$-lactamase enzyme, double disc synergy test (DDST) showed only7 out of 53 isolates $(13.2 \%)$ ) to be positive for metallo- $\beta$-lactamase enzyme whereas combined disc test (CDT) revealed 9 isolates $(16.98 \%)$ to be positive for MBL. Five isolates gave positive result by both CDT and DDST methods whereas 2 isolates showed positive result by DDST only and 4 by CDT only (Table 3 ).

Table 3: Comparison of combined disc test and double disc synergy test

\begin{tabular}{ccc}
\hline Combined disc test (CDT) & Double disc synergy test (DDST) & No. of isolates \\
\hline+ & - & + \\
2 & 5 \\
+ & + & 42 \\
- & - & 53 \\
\hline
\end{tabular}

When screening for AmpC- $\beta$-lactamase producing organism was done by Cefoxitin disc diffusion test, out of 53 MDR isolates, 45 isolates were found to be resistant which were further tested by Disc antagonism test for inducible AmpC and AmpC disc test for plasmid mediated AmpC (Figure 5).

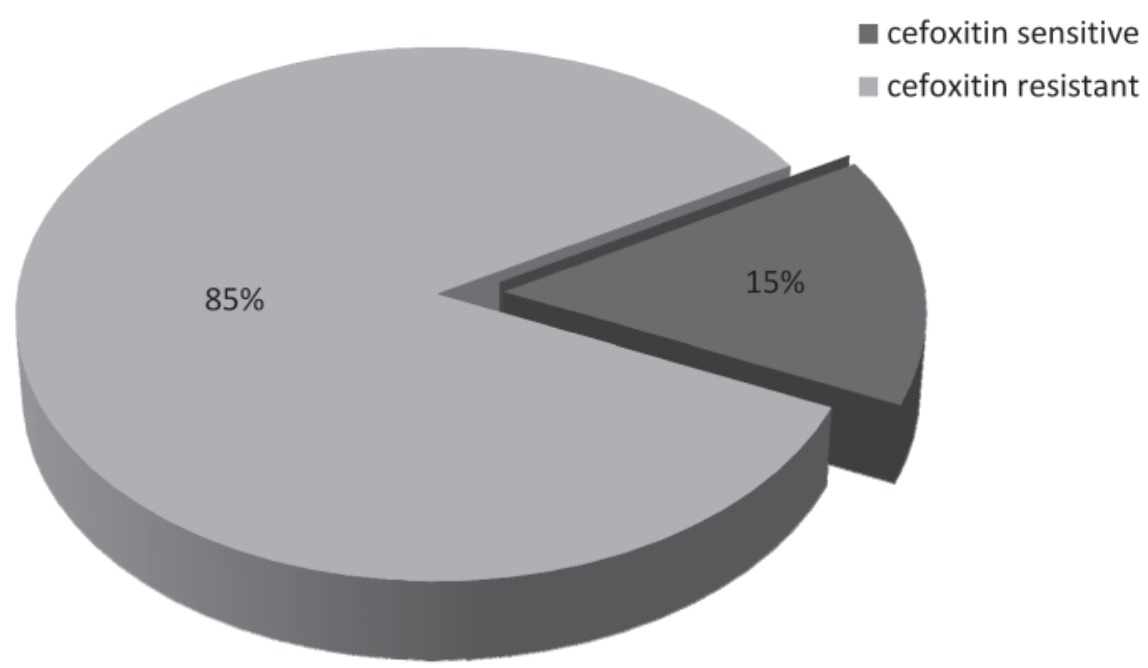

Figure 5: Cefoxitin susceptibility of the MDR isolates 
Out of 45 positively screened MDR isolates, none of them were found to produce chromosomal (inducible) AmpC $\beta$-lactamase whereas $7(15.55 \%)$ were found to produce plasmid mediated (constitutive) AmpC $\beta$ lactamase. Altogether out of $53 \mathrm{MDRPA}$, the prevalence of AmpC producing isolate was found to be $13.2 \%$.
Among the 53 MDRPA strains which were screened phenotypically for various mechanisms of resistance, 20.75\% were MBL producers, 13.2\% AmpC producers, $3.77 \%$ produced both AmpC and MBL and 73.58\% produced neither AmpC nor MBL (Figure 6).



Figure 6: Various mechanisms of resistance among MDR isolates

\section{DISCUSSION}

A total of 756 clinical specimens from the patients of different age groups and wards were included in the study, out of which 75 (9.92\%) of them were identified as $P$. aeruginosa by standard microbiological procedure. A recent study by Khan et al. (2014) reported similar prevalence of $P$. aeruginosa i.e. 6.67\%. Another study by Mohanasoundaram (2011) showed the prevalence rate of $P$. aeruginosa to be $5 \%, 6.8 \%$ and $5 \%$ in three different years which is comparable with our studies. However a study done by Goel et al. (2009) reported a comparatively higher prevalence rate of $P$. aeruginosa (35\%).

The distribution of specimens of $P$. aeruginosa may vary with each hospital as each hospital facility has a different environment associated with it. A study by Chander et al. (2013) showed the major source of $P$. aeruginosa pus/wound (27.6\%) followed by sputum $(24.1 \%)$, urine $(20.7 \%)$, tracheal aspirate $(10.35 \%)$, CVP catheter tube (3.45\%), and 3.45\% from each of BAL fluid, bile, catheter, and high vaginal swab. In another study by Basak et al. (2009), the clinical specimen following $P$. aeruginosa infections were most commonly from urinary tract infection followed by wound infections. However a study done by Mohanasoundaram (2011) showed the maximum no. of isolates from pus and endotracheal secretions and Khan et al. (2014) in his study reported the isolation of maximum no. of $P$. aeruginosa from tracheal secretion and pus. In our study, maximum no. of isolates were obtained from endotracheal secretions $(69.3 \%)$ followed by the category others $(12 \%)$, sputum $(8 \%)$, urine $(5.3 \%)$, blood $(4 \%)$ and pus $(1.3 \%)$.

Increasing resistance to different anti-pseudomonal drugs particularly among hospital strains has been reported world-wide. In this study, the isolates showed highest resistance to Ceftazidime (88\%) followed by Gentamicin (84\%). The isolates resistant to Ciprofloxacin were $78.6 \%$. These strains also showed resistance to carbapenems like Imipenem (24\%) and Meropenem (69.3\%), the precious weapon against $P$. aeruginosa infections (even against MDR isolates) which is an alarming sign. Imipenem was found to be the most effective drug with susceptibility of $76 \%$ followed by Piperacillin/Tazobactam (74.7\%), Piperacillin (41.3\%) and Meropenem (30.7\%). Piperacillin alone tested showed a resistance rate of $58.7 \%$ in this study 
whereas beta-lactam/beta-lactamase inhibitor drug Pipercillin/Tazobactam showed a lower resistance of $25.3 \%$ only, indicating beta-lactamase inhibitor markedly expands the spectrum of activity of betalactams, which makes the combination drug the preferred choice against $P$. aeruginosa infections. A recent study by mohanasaundaram (2011) with 193 P. aeruginosa showed $79 \%$ resistance to Gentamicin followed by Ceftriaxone (75\%), Ciprofloxacin (73\%), Ceftazidime (63\%), Piperacillin (44\%) and Amikacin (41.5\%) which correlates with our study. They also reported resistance to Imipenem (3.7\%) which is less compared to the present study. A study done by Landman et al. (2007) reported resistance of $24 \%$ to imipenem which exactly correlates with our study. In another study by Mohanasoundaram (2011), the resistance rate to imipenem was $27 \%$ in 2009 and $16 \%$ in 2010 which is comparable with this study. Ceftriaxone and ceftazidime are commonest $3^{\text {rd }}$ generation antibiotics in hospital protocols. In our study, the rate of aminoglycoside resistance was also found to be relatively high (Amikacin-73.3\% and gentamicin $84 \%)$. This study shows that the clinical isolates of $P$. aeruginosa are becoming resistant to commonly used antibiotics and gaining more and more resistance to newer antibiotics.

A total of 53 out of 75 isolates were found to be multidrug resistant in this study with the prevalence rate of MDRPA to be $70.6 \%$. Moniri et al. (2005) reported a prevalence rate of $73.9 \%$ of MDRPA. A study by Mahmoud et al. (2012) and Ahmed et al. (2013) reported the prevalence of MDRPA to be 52\% and 63.2\% respectively which is also comparable to our study.

In this study, it was found that $92.45 \%$ of the total MDRPA was isolated from inpatients whereas only $7.54 \%$ belonged to outpatient. As observed in the study, the multidrug resistant strains of $P$. aeruginosa were mainly obtained from inpatients which might be due to increasing invasive procedures that are required for diagnosis and chemotherapy and predispose patients to acquire nosocomial infections with such pathogen. Ahmed et al. (2013) also mentioned the high prevalence of multi-drug resistant $P$. aeruginosa in inpatient than outpatient.

A study by Aggarwal et al. (2008) showed the major source of MDRPA were from sputum and tracheostomy specimen $(28.57 \%)$, followed by pus $(24.13 \%)$, urine $(19.04 \%)$, cerebrospinal fluid and other sterile body fluids (15.38\%) and blood (7.14\%). In a study by Shashikala et al. (2006), 27.6\% of carbapenem resistant $P$. aeruginosa were obtained from surgical wounds and $20.7 \%$ were from endotracheal aspirate showing indwelling devices as major risk factors for the development of resistance. Present study revealed the major source of MDRPA to be endotracheal secrtions (73.58\%) followed by the category others(9.43\%), sputum $(7.54 \%)$, urine $(5.66 \%)$ and $1.88 \%$ each from blood and pus.

The numerous $\beta$-lactamases are encoded either by the chromosomal genes or by the transferable genes which are located on the plasmids or the transposons. Among various mechanisms of resistance, AmpC and metallo- $\beta$-lactamase were detected in this study which is very effective in degrading the anti-pseudomonal agents these days. In 2002 from India, Navaneeth et al. reported MBL production in P. aeruginosa to be $12 \%$. In the present study, we found $20.75 \%$ of MDRPA to be MBL producers. A recent study by Varaiya et al. (2008) showed $20.8 \%$ of MDRPA to be MBL producers. Ugargol (2012) in his study reported $18.8 \%$ of the isolates to be positive for MBL. Similarly, Navaneeth et al. (2002) and Willmann et al. (2013) respectively reported $18 \%$ and $15.9 \%$ of the MDRPA had the ability to produce MBL. Pandya (2011) showed MBL prevalence of $30 \%$.

Since there is no standard guideline for detection of MBL production, different studies have reported to have used different methods. Two different phenotypic methods were used for the detection of metallo- $\beta$ lactamase in our study; Combined disc test (CDT) and Double disc synergy test (DDST). Combined disc test revealed 9 out of 53 i.e. $16.98 \%$ of the MDRPA isolates as MBL positive whereas Double disc synergy test showed only 7 out of 53 i.e. $13.2 \%$ as MBL producers. Five isolates gave positive result by both methods. In this study, both the combinations simultaneously detected 11 isolates i.e. $20.75 \%$ to be positive for MBL. In the evaluation of two selected MBL phenotypic assays, CDT was found to be more sensitive $(16.98 \%)$, than DDST (13.2\%). These results correlated with studies by Behera et al. (2008) (CDT- 88.8\% and DDST-57.14\%), and Pandya et al. (2011) (CDT-96.3\%, DDST- 81.48\%) both of which showed higher susceptibility of CDT than DDST although prevalence rate differs widely from our study. However, it differs from studies by Picao et al. at Brazil from January-December 2005 (CDT- 80\%, DDST-82.6\%) who showed DDST to be a 
more sensitive method than CDT.

Out of 53 MDR isolates, 8 (15\%) were found to be sensitive to cefoxitin whereas 45 of them i.e. $85 \%$ were resistant. A study done by Upadhyaya et al. (2010) reported 196 out of 202 isolates (97\%) to be cefoxitin resistance which is comparable to our study. Hassan et al. (2011) in their study reported $57.33 \%(86 / 150)$ to be cefoxitin resistant. Similarly, another study by Behera et al. (2008) found $78.2 \%$ of the isolates to be cefoxitin resistant.

In our study, the overall prevalence of AmpC was found to be $13.2 \%$ which is comparable to study done by Basak et al. in 2009 (19.3\%). Our study was also comparable with the reports from Aligarh by Shahid et al. in 2004 as $20 \%$ and from Kolkata by Arora et al. in 2005 as $17.3 \%$ and from Varanasi as 22\%. Also, Ugargol (2012) in his study reported $12.8 \%$ of the isolates to be AmpC producer. However, Upadhyaya et al. (2010) reported a high prevalence of $59.4 \%$ of AmpC producing isolates out of which $7 \%$ were positive for inducible and 52.4\% for non-inducible AmpC.

Co-production of AmpC and MBL was found in 3.77\% of the MDRPA isolates in this study. Both of these isolates were from ICU and were resistant to imipenem. Singhal et al. (2005) showed the co-production of AmpC and MBL in $11 \%$ of the isolates. However, Upadhyaya et al. (2010) reported the co-production of AmpC and MBL in $46.6 \%$ of the isolates. Behera et al. (2008) showed the co-production of AmpC and MBL in $48.5 \%$ of the isolates. Both of these findings are comparatively much higher than our study. Production of multiple $\beta$-lactamases by $P$. aeruginosa has tremendous therapeutic consequences and there is a need for urgent action to control the spread of resistant strains. In our study, 39 MDRPA isolates were found to produce neither AmpC nor MBL. These strains may be harboring some other resistance mechanisms like biofilm formation or through virulence factors.

\section{REFERENCES}

Aggarwal R, Chaudhary U and Bala K (2008) Detection of extended spectrum $\beta$-lactamase in $P$. aeruginosa. Indian J Path Microbiol 51(2): 222-224.

Ahmed S and Al-Harbi MN (2013) Antibiotic susceptibility pattern of isolates of $P$. aeruginosain a Saudi Arabian Hospital. Bangladesh J Med Sci 13(1): 45-48.

Arora S and Bal M (2005) AmpC $\beta$-lactamase producing bacterial isolates from Kolkata hospital. Indian J Med Res 122(3): 224.

Basak S, Khodke M, Bose S and Mallick SK (2009) Inducible Amp C $\beta$-lactamase producing $P$. aeruginosa isolated in a rural hospital of central India. J ClinDiagn Res 3: 1921-1927.

Behera B, Mathur P, Das A, Kapil A and Sharma V (2008) An evaluation of four different phenotypic techniques for detection of metallo- $\beta$-lactamase producing $P$. aeruginosa. Indian J Med Microbiol 26(3): 233-237.

Chander A and Raza MS (2013) Antimicrobial susceptibility patterns of $P$. aeruginosa clinical isolates at a tertiary care hospital in kathmandu, Nepal.Asian J Pharm Clin Res 6(3): 235-238

Clinical and Laboratory Standard Institute (CLSI) (2013) M100-S16

Goel N, Chaudhari U, Aggrawal R and Bala K (2009). Antibiotic susceptibility pattern of Gram negative bacilli isolated from the lower respiratory tract of ventilated patients in the intensive care unit. Indian J Crit Care Med 13(3): 148-150.

Hassan A, Usman J, Kaleem F, Omair M, Khalid A and Iqbal M (2011) Evaluation of different detection methods of biofilm formation in theclinical isolates. Braz J Infect Dis 15(4): 305-311.

Ishii Y, Eto M, Mano Y, Tateda K and Yamaguchi K (2010) In vitro potentiation of carbapenems with ME1071, a novel metallo- $\beta$-lactamase inhibitor, against metallo- $\beta$-lactamase-producing $P$. aeruginosa clinical isolates. Antimicrob Agents Chemother 54(9): 3625- 3629.

Kalaivani R (2011) Antibiotic resistance mechanisms in clinical isolates of $P$. aeruginosa in a tertiary care hospital (Doctoral Dissertation, Pondicherry University).

Kapoor R, Wadman MW, Dohm MT, Czyzewski AM, Spormann AM and Barron AE (2011) Antimicrobial peptoids are effective against $P$. aeruginosa biofilms. Antimicrob Agents Chemother 55(6): 3054-3057.

Khan J, Wahab A, Qayyum A and Jamshed S (2014) Drug resistance pattern of $P$. aeruginosa isolates at PIMS Hospital, Islamabad, Pakistan. J Chem Pharm $\operatorname{Res}$ 6(11): 715-719. 
Landman D, Bratu S, Kochar S, Panwar M, Trehan M, Doymaz M and Quale J (2007) Evalution of antimicrobial resistance among $P$. aeruginosa, Acinetobacter baumannii and Klebsiella pneumoniae in Brooklyn, NY. J Antimicrob Chemother 60(1): 7882.

Martinez LR, Jimenez LL, Ostuni V, Fuste E, Vinuesa T and Vinas M (2011) A mechanism of carbapenem resistance due to a new insertion element (ISPa133) in P. aeruginosa. Int Microbiol 14: 51-58.

Mohanasoundaram KM (2011) The Antimicrobial resistance pattern in the clinical isolates of $P$. aeruginosa in a tertiary care hospital; 2008-2010 (A 3 Year Study). J Clin Diag Res 5(3): 491-494.

Moniri R, Mosayebi Z, Movahedian AH and Mousavi GA (2005) Emergence of multi-drug-resistant $P$. aeruginosa isolates in neonatal septicemia. J Infect Dis Antimicrob Agents 22(2): 39-44.

Navaneeth BV, Sridaran D, SahavD and Belwadi MR (2002) A preliminary study on MBL producing $P$. aeruginosa in hospitalized patient. Indian J Med Res 116: 264-267.

Pandya N (2011) Evaluation of various methods for MBL detection in Gram negative bacilli. Int J Biol Med Ress 2(3): 775-777.

Picao RC, Andrade SS, Nicoletti AG, Campana EH, Moraes GC, Mendes RE and Gales AC (2008) Metallo- $\beta$-lactamase detection: comparative evaluation of double-disc synergy versus combined disc tests for IMP-, GIM-, SIM-, SPM-, or VIM-producing isolates. J Clin Microbiol 46(6): 2028-2037

Shahid S, Malik A, Agarwal M, Singhal S (2004) Phenotypic detection of extended spectrum and AmpC $\beta$-lactamases by a new spot inoculation method and modified three dimensional extract test: comparison with the three dimensional extract test. J Antimicrob. Chemother 54: 684-687.

Shanthi M and Sekar U (2009) Multi-drug resistant $P$. aeruginosa and Acinetobacter baumannii infections among hospitalized patients: risk factors and outcomes.J Assoc Physicians India 57: 636-638
Shashikala, Kanungo R, Srinivasan S and Devi S (2006) Emerging resistance to carbapenem in hospital acquired Pseudomonas infection: A case of concern. Indian J Pharmacol 38: 287-290.

Singhal S, Mathur T, Khan S, Upadhyay DJ and Chugh S (2005) Evaluation of methods for AmpC $\beta$-lactamase in gram negative clinical isolates from tertiary care hospitals. Ind J Med Microbiol 23: 120-124.

Tian GB, Adams-Haduch JM, Bogdanovich T, Wang HN and Doi Y (2011) PME-1, an extended-spectrum $\beta$-lactamase identified in $P$. aeruginosa. Antimicrob Agents Chemother 55(6): 2710-2713.

Tsakris A, Poulou A, Kristo I, Pittaras T, Spanakis N, Pournaras S and Markou F (2009) Large dissemination of VIM- 2- metallo- $\beta$-lactamaseproducing $P$. aeruginosa Strains Causing Health Care-Associated Community-Onset Infections. J Clin Microbiol 47: 3524-3529.

Ugargol AR (2012) Characterization, Antibiotic resistance pattern and detection of Metallo- $\beta$ lactamases and AmpC in P. aeruginosa in a tertiary care hospital (Doctoral Dissertation, Rajiv Gandhi University of Health Sciences)

Upadhyay S, Sen MR and Bhattacharjee A (2010) Presence of different $\beta$-lactamase classes among clinical isolates of P. aeruginosa expressing AmpC $\beta$-lactamase enzyme.J Infect Dev Ctries4(4): 239-242.

Varaiya A, Kulkarni N, Kulkarni M, Bhalekar P and Dogra J (2008) Incidence of metallo $\beta$ lactamase producing $P$. aeruginosa in ICU patients. Indian J Med Res 127: 398-402.

Willmann M, Kuebart I, Marschal M, Schroppel K, Vogel W, Flesch I and Peter S (2013) Effect of metallo- $\beta$ lactamase production and multidrug resistance on clinical outcomes in patients with $P$. aeruginosa bloodstream infection: a retrospective cohort study. BMC Infect Dis 13(1): 515.

Yousefi S, Farajniaa S, Nahaeib MR, Akhic MT, Ghotaslouc R, Soroushc MH, Naghilid B and Jazanie NH (2010) Detection of metallo- $\beta$ lactamase-encoding genes among clinical isolates of $P$. aeruginosa in northwest of Iran. Diagn Microbiol Infect Dis 68: 322-325. 